\title{
Dispersion of ventricular repolarisation: a marker of ventricular arrhythmias in patients with previous myocardial infarction
}

\author{
M Zaidi, A Robert, R Fesler, C Derwael, C Brohet
}

\begin{abstract}
Objective-To examine whether, in coronary patients after myocardial infarction, the dispersion of ventricular repolarisation measured through QT and JT intervals from a surface electrocardiogram could allow separation of those with ventricular tachyarrhythmias (VT) complicating their myocardial infarct from those without.

Design-A retrospective comparative study.

Setting-University hospital.

Patients-39 patients with myocardial infarction complicated by VT, 300 patients after myocardial infarction without arrhythmic events, and 1000 normal subjects. The myocardial infarction groups were divided into anterior, inferior, and mixed locations.

Interventions-A computer algorithm examined an averaged cycle from a 10 second record of 15 simultaneous leads (12 lead ECG + Frank XYZ leads). After interactive editing, four intervals were computed: QTapex, JTapex, QTend, and JTend. For each interval, the dispersion was defined as the difference between the maximum and minimum values across the 15 leads.
\end{abstract}

Results-The mean values of all four dispersion indices were higher in patients with myocardial infarction than in normal subjects $(p<0.01)$. In the infarct groups, patients with VT had significantly greater mean and centile dispersion values than those without VT. For instance, the 97.5th centile value of QTend was $65 \mathrm{~ms}$ in normal individuals, $90 \mathrm{~ms}$ in infarct patients without arrhythmia, and $128 \mathrm{~ms}$ in those with VT; $70 \%$ of the infarct patients who developed serious ventricular arrhythmias had values exceeding the 97.5th centile of the normal group, while only $18 \%$ of the infarct patients without arrhythmia had dispersion values above this normal upper limit. Among the infarct patients, nearly half of those (18 of 39) with tachyarrhythmias had dispersion values that exceeded the 97.5 th centile of those without arrhythmia.

Conclusions-Dispersion of ventricular repolarisation may be a good non-invasive tool for discriminating coronary patients susceptible to VT from those who are at low risk.

(Heart 1997;78:371-375)
Keywords: QT dispersion; myocardial infarction; computer analysis; arrhythmias

The dispersion of ventricular repolarisation, defined as the QT interval variability from lead to lead on the surface electrocardiogram (ECG), could reflect a regional heterogeneity of myocardial recovery. ${ }^{1-4}$ Increased dispersion has been reported as a non-invasive marker of an electrophysiological arrhythmogenic substrate and it has been associated with high risk of ventricular arrhythmias and sudden death in various cardiac disorders. ${ }^{5-8}$ In a recent normalisation study, we found that not only QT interval dispersion but also other indices measured on the whole QRS-T complex, especially the JT index, could be used in the quantitative assessment of the dispersion of ventricular repolarisation. ${ }^{9}$ However, there has been only a limited amount of data published on the use of indices other than the QT interval.

Patients with myocardial infarction may have an inhomogeneous ventricular repolarisation process. These patients are at increased risk of developing life threatening ventricular arrhythmias. Indeed, about $10 \%$ of patients who survive to hospital discharge die the first year after the acute event. ${ }^{10}$ One third of the deaths occur suddenly and are presumed to be caused by ventricular arrhythmias. ${ }^{11}$ Since patients are at increased risk of arrhythmic death after myocardial infarction, assessment of dispersion of ventricular repolarisation could have important clinical implications. It would be worthwhile to identify patients at risk by a simple, non-invasive, and low cost procedure so that they could receive appropriate treatment.

We therefore investigated whether the dispersion of ventricular repolarisation, measured through QT and JT intervals from surface electrocardiograms by a new computerised method, might allow us to separate myocardial infarction patients with a documented history of ventricular tachyarrhythmias from those without.

\section{Methods}

STUDY POPULATION

Dispersion of ventricular repolarisation was assessed in 39 patients with myocardial infarction (mean age 59 (SD 10) years; 33 men and six women), complicated by the occurrence of ventricular tachyarrhythmias such as nonsustained $(\mathrm{n}=15)$ or sustained $(\mathrm{n}=12)$ ventricular tachycardia or ventricular fibrillation $(n=12)$. The results of these patients were
Accepted for publication 10 June 1996 


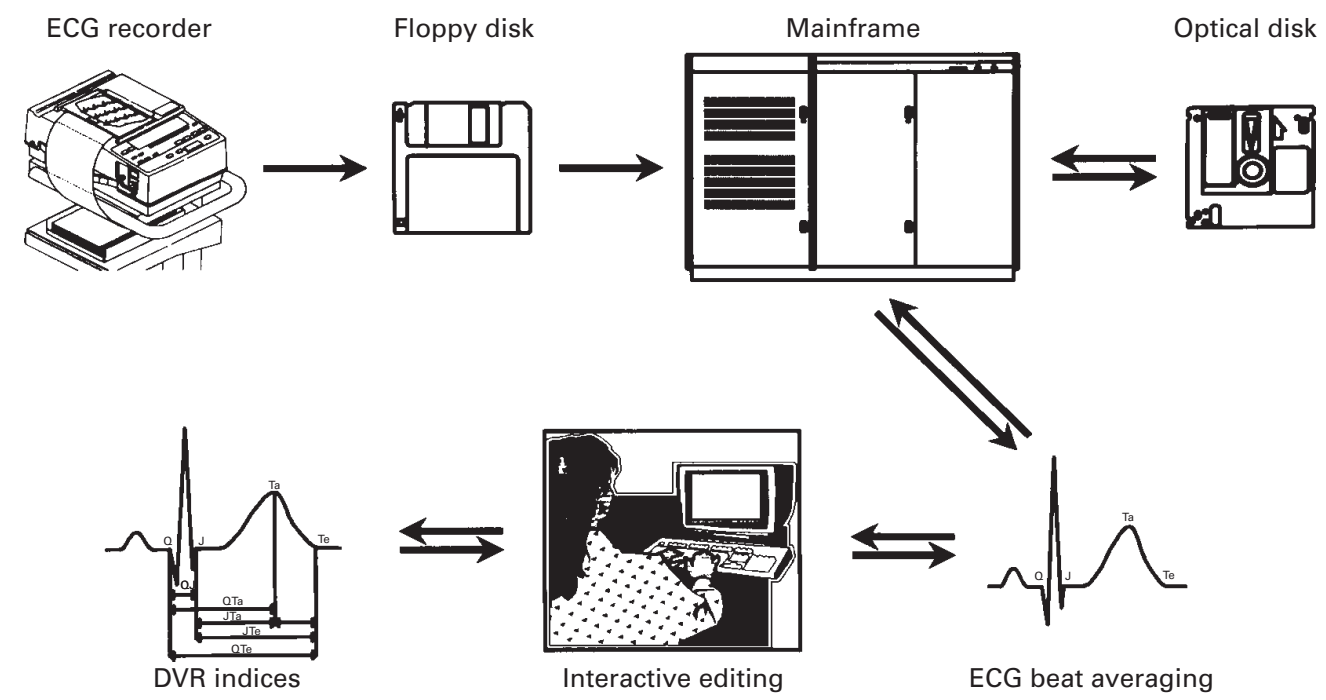

Figure 1 Algorithm flow chart showing the data acquisition system and the semiautomatic assessment of ventricular repolarisation.

compared with those of 300 other patients with myocardial infarction but without any arrhythmic complication (mean age 58 (11) years; 250 men and 50 women). According to the infarction site, the infarct groups were subdivided into three locations: anterior $(\mathrm{n}=8)$, inferior ( $\mathrm{n}$ $=16)$, and mixed $(n=15)$. The control group consisted of 1000 healthy subjects (mean age 46 (16) years; 410 men and 590 women) with no structural cardiac abnormalities. These normal controls were selected on the basis of history, physical examination, chest $x$ ray and, in some cases, echocardiography. Most were ambulatory subjects seen at the outpatient clinic for systematic health checks. A few of them were hospital inpatients undergoing a prospective work up before gynaecological or orthopaedic surgery. All electrocardiograms of coronary patients were recorded after the acute phase of myocardial infarction, with a delay ranging from two months to 22 years (mean 55 (72) months).

\section{PROCEDURES}

The electrocardiograms were recorded on a machine capable of simultaneous 15 lead acquisition: the 12 standard ECG leads and the three orthogonal Frank XYZ leads (Marquette Electronics, Milwaukee, Wisconsin, USA). Each record consisted of a $10 \mathrm{~s}$ digitised 15 lead sample at $500 \mathrm{~Hz}$ with a resolution of $5 \mathrm{mV}$. The electrocardiographic data were transferred on disk to a computer where beat averaging of each record was performed by a special program (fig 1). Several fiducial points were then automatically identified on the resulting averaged P-QRS-T complex: QRS onset $(\mathrm{Q})$ and offset $(\mathrm{J})$ and the $\mathrm{T}$ wave apex (Ta) and end (Tend). This program defines the J point as the return of the QRS deflection to the isoelectric baseline and the Tend point as the return of the distal limb of the T wave to the TP baseline level. The apex of the $T$ wave is defined as the maximum positive or negative amplitude in a search region starting at $40 \mathrm{~ms}$ after the J point until the end of the $T$ wave. Further adjustment of the fiducial points can be made in each individual lead by manual editing through an interactive method using a graphical display. From the validated fiducial points, four intervals were directly computed and considered for the present study: QTa, JTa, QTend, and JTend (fig 2). When a U wave was present, the two intervals addressing the global ventricular repolarisation were measured from the $Q$ and J points, respectively, to the nadir of the curve joining the $\mathrm{T}$ and $\mathrm{U}$ waves. For each interval, the dispersion was defined as the difference between the maximum and the minimum values across the 15 leads. According to this definition, four dispersion indices were calculated: QTa-d, JTa-d, QTe-d, and JTe-d. All values were corrected for heart rate using Bazett's formula. ${ }^{12}$ In order to minimise interobserver variability, the measurements were performed by the same observer. Extensive clinical validation of this semiautomated method has been undertaken in our laboratory. ${ }^{89}$

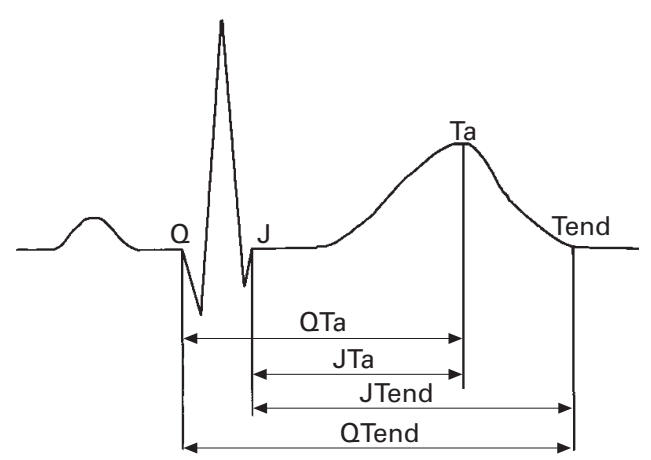

Figure 2 After the validation of the fiducial points on the resulting averaged $P-Q R S-T$ complex, four intervals are automatically computed: QTend from QRS onset $(Q)$ to $T$ wave end (Tend), QTa from QRS onset to T wave apex (Ta), FTend from $\mathcal{F}$ point to $T$ wave end, and $\mathfrak{F}$ Ta from $\mathcal{F}$ point to $T$ wave apex. 
STATISTICAL ANALYSIS

A particular record was excluded from statistical analysis if it contained more than five leads rejected because there was no reliable $T$ wave end location, for example, a flat $\mathrm{T}$ wave, excessive noise level, artefact, and so on. The three groups were compared using analyses of variance with either the Brown-Forsythe test or an $\mathrm{F}$ test according to the significance of Levene's test for equal variances across groups. Any significant result initiated contrast analyses for pairwise comparisons using either separate or pooled $t$ tests with Bonferroni adjusted significance levels. The data are presented as means (SD).

\section{Results}

Table 1 shows the global distribution of the mean values of the four dispersion indices among the three clinical groups. The mean dispersion values were smallest in the normal group, higher in the infarction group without arrhythmic complication ( $p<0.01)$, and highest in infarct patients with a history of ventricular tachyarrhythmias $(p<0.001)$. In all three groups, the values of the JT indices, which represent the dispersion of the sole repolarisation phase, were larger than those of the QT indices, which encompass both depolarisation and repolarisation phases.

Table 2 gives more details on the comparisons of the mean dispersion values and the centile ranges between the three subgroups of infarct patients, divided according to infarct location. Among patients with arrhythmic complications, there were eight anterior, 16 inferior, and 15 mixed myocardial infarcts. In each infarction subgroup, patients with ventricular tachyarrhythmias had significantly higher indices of ventricular repolarisation dispersion than those without. Furthermore, each mean value in the patients with ventricular tachyarrhythmias was close to the corresponding 97.5 th centile value of the patients without such arrhythmias.

Figure 3 shows the distribution of the values of the most commonly used ventricular repolarisation dispersion index, the Qte dispersion, in the three clinical groups. Although the mean

Table 1 Distribution of the four dispersion indices among the three clinical groups

\begin{tabular}{llll}
\hline DVR indices & Normal $(n=1000)$ & MI without VT $(n=300)$ & MI with VT $(n=39)$ \\
\hline JTa-d (ms) & $49(19)$ & $60(24)$ & $88(45)$ \\
JTe-d (ms) & $37(17)$ & $52(21)$ & $87(28)$ \\
QTa-d (ms) & $42(18)$ & $54(25)$ & $81(45)$ \\
QTe-d (ms) & $30(15)$ & $46(20)$ & $80(25)$ \\
\hline
\end{tabular}

Values are mean (SD)

DVR, dispersion of ventricular repolarisation; Normal, normal subjects; MI, myocardial infarction; VT, ventricular tachyarrhythmias. See text for explanation of DVR indices.

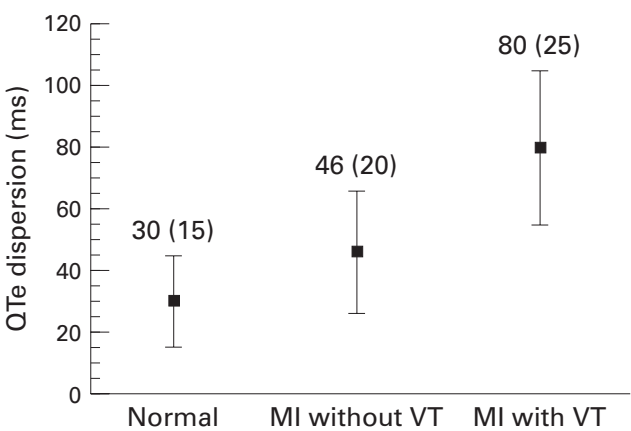

Figure 3 Comparison of QTe dispersion index values (means; bars $=1 S D$ ) highlighting the differences and the overlap between the three clinical groups: normal subjects, patients with myocardial infarction (MI) without ventricular tachyarrhythmia (VT), and patients with myocardial infarction complicated by arrhythmias.

values differ significantly between the groups, there was an important overlap between the distributions of individual values of QTe-d in the normal subjects and in the patients after myocardial infarction, although in the latter this overlap was less marked between those with and those without ventricular tachyarrhythmias. For instance, the 97.5th centile value of QTe-d was $65 \mathrm{~ms}$ in normal subjects, $90 \mathrm{~ms}$ in the group without arrhythmias, and $128 \mathrm{~ms}$ in patients with ventricular tachyarrhythmias after myocardial infarction.

Another way to express the results is to analyse the discriminant power of the QTe dispersion, which can be represented by the percentage of patients in the myocardial infarction groups who have dispersion values exceeding the 97.5th centile of the normal group. Seventy per cent of the infarct patients who developed serious ventricular arrhythmias had values of both QTe-d and JTe-d that exceeded the 97.5th centile of the normal group, while only $18 \%$ and $15 \%$ of the infarct patients without arrhythmic complication had dispersion values of QTe and JTe intervals above this normal upper limit. Moreover, by examining only the two infarct groups, we found that nearly half the patients (18 of 39) with tachyarrhythmias complicating their myocardial infarction had dispersion values that exceeded the 97.5th centile of the patients who had had no arrhythmia.

\section{Discussion}

It is well established that ventricular repolarisation characteristics play an important role in arrhythmogenesis. ${ }^{13-15}$ In the assessment of ventricular repolarisation, prolongation of the QT interval duration is known to contribute to triggering of arrhythmias. ${ }^{216}$ Recently, the dispersion of ventricular repolarisation, measured

Table 2 Comparisons of the mean dispersion values and the centile ranges between the patients with myocardial infarction divided into three subgroups according to the infarct locations

\begin{tabular}{|c|c|c|c|c|c|c|}
\hline \multirow[b]{2}{*}{ DVR indices } & \multicolumn{2}{|c|}{ Anterior myocardial infarction } & \multicolumn{2}{|c|}{ Inferior myocardial infarction } & \multicolumn{2}{|c|}{ Mixed myocardial infarction } \\
\hline & With VT $(n=8)$ & Without VT $(n=100)$ & With VT $(n=16)$ & Without VT $(n=100)$ & With VT $(n=15)$ & Without VT $(n=100)$ \\
\hline JTe-d (ms) & 98 (19) $(75,131)$ & $57(21)(21,100)$ & $86(28)(36,153)$ & $46(20)(19,86)$ & $82(32)(27,126)$ & $52(22)(19,94)$ \\
\hline QTe-d (ms) & $89(17)(58,110)$ & $51(20)(13,90)$ & $78(24)(44,128)$ & $40(20)(12,85)$ & 77 (29) $(27,109)$ & $47(21)(14,87)$ \\
\hline
\end{tabular}

Values are mean $(\mathrm{SD})$ and $(2.5,97.5)$ centiles.

DVR, dispersion of ventricular repolarisation; VT, ventricular tachyarrhythmias. See text for explanation of DVR indices. 
from the surface ECG and defined as the interlead variability of the QT interval, has been reported as a new method for analysing ventricular repolarisation. Dispersion of repolarisation is thought to reflect regional heterogeneity of the recovery process within the myocardium, which is believed to be important in the genesis of ventricular arrhythmias. ${ }^{1-4}$ The concept that QT interlead variability reflects the dispersion of ventricular repolarisation is supported by the close correlation between changes in dispersion of repolarisation from ventricular monophasic action potential recordings and changes in QT interval variation produced by ventricular pacing. ${ }^{17-19}$ Furthermore, dispersion of ventricular repolarisation has been shown to be increased in various cardiac disorders known to be complicated by ventricular arrhythmias, such as long QT syndrome, drug toxicity, and dilated and hypertrophic cardiomyopathies. ${ }^{5-8}$

Life threatening ventricular arrhythmias represent a major complication in coronary patients with myocardial infarction. ${ }^{11}{ }^{11}$ In experimental investigations, regional disparities in repolarisation have been measured by electrodes placed several millimetres apart with a small field of view. Variation in ventricular recovery times is an important factor in experimental tachyarrhythmias. The usual site of abnormal dispersion from which arrhythmias occur is at the border zone of the infarcted area. $^{20-22}$ The search for a good indicator of susceptibility to ventricular arrhythmias in patients after myocardial infarction is thus of great importance.

In a previous report on a normalisation study, we described in detail the semiautomated method used for quantifying the dispersion of ventricular repolarisation from 15 lead digitised ECGs. We showed that the incorporation of the three orthogonal XYZ leads as well as the inclusion of the JT interval increased the dispersion differences between several clinical groups. ${ }^{9}$ This method was applied here to the study of repolarisation dispersion after myocardial infarction with and without arrhythmic complications, compared with normal controls. The results of our study show that QT and JT dispersion indices, measured as the maximum difference of each interval on a surface ECG, are significantly increased in patients after myocardial infarction complicated by ventricular tachyarrhythmias compared with those who had no arrhythmic event.

In a recent study, Van de Loo et al measured QT dispersion in 127 subjects, including 50 without structural heart disease and 77 with myocardial infarction. They found that the mean (SD) values of QT dispersion were 30 (10) $\mathrm{ms}$ in normal controls, 56 (24) $\mathrm{ms}$ in patients with myocardial infarction, and 88 (30) $\mathrm{ms}$ in patients with ventricular fibrillation after myocardial infarction. ${ }^{23}$ Our results are consistent with those findings. Indeed, we found nearly the same mean QT dispersion values - 30 (15) ms in healthy subjects, 46 (20) $\mathrm{ms}$ in patients after myocardial infarction, and 80 (25) $\mathrm{ms}$ in patients with ventricular tachyarrhythmias occurring after myocardial infarc- tion. Higham et al, in a prospective study of 55 coronary patients, found that QT dispersion was significantly increased in patients with myocardial infarction compared with those admitted for unstable angina, at 69 (18) $v 38$ (13) ms. They also showed that QT dispersion values were greater in four postinfarction patients who developed ventricular fibrillation than in those without ventricular arrhythmias after acute myocardial infarction (87 (15) $v 66$ (18) $\mathrm{ms}) .{ }^{24}$ The dispersion values of these four patients were very similar to those found in our group of 39 postinfarction patients with ventricular tachyarrhythmias. In an investigation by Pye et al, ${ }^{25} \mathrm{QT}$ dispersion was markedly increased in myocardial infarct patients with ventricular tachyarrhythmias in comparison with a control group, at 82 (22) v 38 (10) ms, and in a retrospective study, Glancy et al reported that increased QT dispersion measured on ECGs recorded four weeks after myocardial infarction was associated with increased mortality during the next five years. ${ }^{26}$

Our results are also in agreement with those of Perkiomaki and coworkers, who examined the value of QT dispersion from 12 lead ECGs in patients with previous myocardial infarction with and without a history of serious arrhythmic events or inducible ventricular tachycardia during programmed electrical stimulation. ${ }^{27}$ They found that QT dispersion was significantly increased in patients with susceptibility to ventricular tachyarrhythmias compared with healthy subjects or postinfarction patients with no susceptibility to arrhythmias. Multivariate analysis showed that QT dispersion was the independent factor that most effectively identified the patient groups with and without susceptibility to ventricular tachyarrhythmias.

Zareba et al reported that increased dispersion of ventricular repolarisation was associated with a greater likelihood of arrhythmic cardiac death and contributed independently to the risk of sudden death in patients with coronary artery disease. ${ }^{6}$ They evaluated repolarisation dispersion not only from the QT interval but also from the JT interval, and they found that JT dispersion was increased in postinfarction patients who died suddenly as compared with survivors (82 (26) v 59 (20) $\mathrm{ms})$. Their results are very similar to ours, especially for the new JT dispersion index, which was 87 (28) $\mathrm{ms}$ in patients with ventricular tachyarrhythmias and 52 (21) $\mathrm{ms}$ in the infarct patients without arrhythmic complication. This was the first published study in which dispersion of ventricular repolarisation was assessed by means of the JT interval, disregarding the QRS duration. This concept is closer to reflecting the sole recovery process than the most commonly used dispersion index, the QT interval, which encompasses both depolarisation and repolarisation phases.

The results of our study provide more evidence of the value of measuring dispersion of ventricular repolarisation on the surface ECG to identify susceptibility to arrhythmias. We are well aware that our group of patients with ventricular tachyarrhythmias is not homogeneous, since it was composed of patients with 
ventricular fibrillation and sustained and nonsustained ventricular tachycardia. The electrophysiological basis of these different kinds of arrhythmia is not the same, and one can postulate that within this group there might be important variations in dispersion features. However, because of the limited sample size, we did not attempt to differentiate between the three types of ventricular tachyarrhythmia.

Along with late potentials and heart rate variability, dispersion of ventricular repolarisation might be used to select the patients in whom more expensive and invasive investigations, such as programmed ventricular stimulation, are indicated. It was interesting to find that differentiation between the two infarct groups on the basis of their repolarisation dispersion remained possible many months after the acute event. We have also confirmed that JT dispersion, which represents the sole repolarisation process, is a valuable index in the assessment of repolarisation dispersion. These findings suggest that the use of a single value of the QT interval on the surface ECG might not be sufficient. Far more information about ventricular repolarisation can be obtained by calculating the dispersion of each interval.

The dispersion of ventricular repolarisation seems to be a good non-invasive tool for discriminating patients susceptible to ventricular tachyarrhythmias from those who are at low risk. The real accuracy of this method compared with others should of course be established. Because of its retrospective nature, our study does not provide information about the predictive value of the dispersion of ventricular repolarisation for future arrhythmic events in individual patients after myocardial infarction. It simply illustrates the gradual increase in the spatial heterogeneity of ventricular recovery from normal subjects to patients with uncomplicated myocardial infarction to those with serious ventricular arrhythmias.

1 Kuo CS, Munakata K, Reddy CD, Surawicz B. Characteristics and possible mechanism of ventricular arrhythmia dependent on the dispersion of action potential durations. Circulation 1983;67:1356-67.

2 Surawicz B. The QT interval and cardiac arrhythmias. Annu Rev Med 1987;38:81-9.

3 Day CP, McComb JM, Campbell RWF. QT dispersion: an indication of arrhythmic risk in patients with long QT intervals. Br Heart $\mathcal{7} 1990 ; 63: 342-4$.

4 Higham PD, Hilton CJ, Aitcheson JD, Furniss SS, Bourke JP, Campbell RWF. QT dispersion does reflect regional variation in ventricular recovery. Circulation 1992, 86(suppl): 392 .
5 Linker NJ, Colonna P, Kekwick CA. Assessment of QT dispersion in symptomatic patients with the congenital long QT syndromes. Am $\mathcal{f}$ Cardiol 1992;69:634-8.

6 Zareba W, Moss AJ, le Cessie S. Dispersion of ventricular repolarization and arrhythmic cardiac death in coronary artery disease. Am f Cardiol 1994;74:550-3.

7 Hii JTY, Wyse DG, Gillis AM, Duff HJ, Solylo MA, Mitchell CB. Precordial QT interval dispersion as a marker of torsade de pointes. Circulation 1992;86:1376-82.

8 Zaidi M, Robert A, Fesler R, Derwael C, Brohet C. Dispersion of ventricular repolarization in hypertrophic cardiomyopathy. F Electrocardiol 1996;29(suppl):28-33.

9 Zaidi M, Robert A, Fesler R, Derwael C, De Kock M, Brohet C. Computer-assisted study of the ECG indices of the dispersion of ventricular repolarization. $\mathcal{F}$ Electrocardiol 1996;29:199-211.

10 The Multicentric Postinfarction Research Group. Risk stratification and survival after myocardial infarction. $N$ Engl F Med 1983;309:331-6.

11 Launbjerg J, Wolf MD. QT interval prolongation as predictor of sudden death in patients with myocardial infarction. Circulation 1978;57:1074-7.

12 Bazett HC. An analysis of the time relations of electrocardiograms. Heart 1920;7:353-70.

13 Merx W, Yoon MS, Han J. The role of local disparity in conduction and recovery time on ventricular vulnerability to fibrillation. Am Heart f 1977;94:603-10.

14 Schwartz PJ, Wolf S. QT interval prolongation as predictor of sudden death in patients with myocardial infarction. Circulation 1978;57:1074-7.

15 Mirvis DM. Spatial variation of QT intervals in normal persons and in patients with acute myocardial infarction. $\mathcal{f} \mathrm{Am}$ Coll Cardiol 1985;5:625-31.

16 Schwartz PJ. Idiopathic long QT syndrome: progress and questions. Am Heart f 1985;109:399-411.

17 Higham PD, Hilton CJ, Griffiths CJ, Furniss CS, Campbell RWF. Relation between QT intervals and epicardial monophasic action potentials [abstract]. Br Heart $\mathcal{f}$ 1992;63:123.

18 Day CP, McComb J, Campbell RWF. QT dispersion in sinus beats and ventricular extrasystoles in normal hearts. Br Heart f 1992;67:39-41.

19 Morgan JM, Cunningham D, Rowland E. Dispersion of monophasic action potential duration: demonstrable in humans after premature ventricular stimulation but not in steady state. F Am Coll Cardiol 1992;19:1244-53.

20 Gough WB, Mehra R, Restivo M, Zeller RH, El-Sherif N. Reentrant ventricular arrhythmias in the late myocardial infarction period in the dog. Correlation of activation and refractory maps. Circ Res 1985;57:432-42.

21 Battle WE, Naimi S, Avitall B, Brilla AH, Banas JS, Bete $\mathrm{SM}$, et al. Distinctive time course of ventricular vulnerability to fibrillation during and after release of coronary ligation. Am f Cardiol 1974;34:42-7.

22 Surawicz B. Dispersion of refractoriness in ventricular arrhythmias. In: Zipes DP, Jalife J, eds. Cardiac electrophysiology: from cell to bedside. Philadelphia: WB Saunders, 1990:377-85.

23 Van de Loo A, Arendts W, Hohnloser SH. Variability of QT dispersion measurements in the surface electrocardiogram
in patients with acute myocardial infarction and in normal subjects. Am f Cardiol 1994;74:1113-18.

24 Higham PD, Furniss SS, Campbell RWF. QT dispersion and components of the QT interval in ischaemia and infarction. Br Heart f 1995;73:32-6.

25 Pye M, Quinn AC, Cobbe SM. QT interval dispersion: a non-invasive marker of susceptibility to arrhythmia in patients with sustained ventricular arrhythmias? Br Heart $\mathcal{F}$ 1994;71:511-14.

26 Glancy JM, Garratt CJ, Woods KL, de Bono DP. QT dispersion and mortality after myocardial infarction. Lancet 1995;345:945-8.

27 Perkiomaki JS, Koistinen MJ, Yli-Mayry S, Huikuri HV. Dispersion of QT interval in patients with and without susceptibility to ventricular tachyarrhythmias after previous myocardial infarction. $\mathcal{F}$ Am Coll Cardiol 1995;26:174-9. 